\title{
Right-sided infective endocarditis in association with a left-to-right shunt complicated by haemoptysis and acute renal failure: a case report
}

Rubi Stephani Hellwege* ${ }^{*}$ and Meinrad Gawaz

\begin{abstract}
Background: Infective endocarditis has a relevant clinical impact due to its high morbidity and mortality rates. Rightsided endocarditis has lower complication rates than left-sided endocarditis. Common complications are multiple septic pulmonary embolisms, haemoptysis, and acute renal failure. Risk factors associated with right-sided infective endocarditis are commonly related to intravenous drug abuse, central venous catheters, or infections due to implantable cardiac devices. However, patients with congenital ventricular septal defects might be at high risk of endocarditis and haemodynamic complications.
\end{abstract}

Case presentation: In the following, we present the case of a 23-year-old man without a previous intravenous drug history with tricuspid valve Staphylococcus aureus endocarditis complicated by acute renal failure and haemoptysis caused by multiple pulmonary emboli. In most cases, right-sided endocarditis is associated with several common risk factors, such as intravenous drug abuse, a central venous catheter, or infections due to implantable cardiac devices. In this case, we found a small perimembranous ventricular septal defect corresponding to a type 2 Gerbode defect. This finding raised the suspicion of a congenital ventricular septal defect complicated by a postendocarditis aneurysmal transformation.

Conclusions: Management of the complications of right-sided infective endocarditis requires a multidisciplinary approach. Echocardiographic approaches should include screening for ventricular septal defects in patients without common risk factors for tricuspid valve endocarditis. Patients with undiagnosed congenital ventricular septal defects are at high risk of infective endocarditis. Therefore, endocarditis prophylaxis after dental procedures and/or softtissue infections is highly recommended. An acquired ventricular septal defect is a very rare complication of infective endocarditis. Surgical management of small ventricular septal defects without haemodynamic significance is still controversial.

Keywords: Case report, Right-sided endocarditis, Ventricular septal defect, Gerbode defect, Tricuspid valve, Staphylococcus aureus, Glomerulonephritis, Haemoptysis, Septic pulmonary embolisms

${ }^{*}$ Correspondence: Stephani.Hellwege@med.uni-tuebingen.de; shellwege@outlook.com

Department of Cardiology and Angiology, University Hospital, University of Tübingen, Tübingen, Germany

\section{Background}

The diagnosis and management of patients with infective endocarditis (IE) require extensive clinical assessment, advanced cardiac imaging, and an interdisciplinary approach to decrease morbidity and mortality. Rightsided IE has a lower prevalence (10-15\%) with lower 
complication rates than left-sided endocarditis $[1,2]$. In most cases, Staphylococcus aureus is identified as the pathogen in blood cultures, and the management is conservative with specific antibiotic therapy $[1,2]$. Common complications of right-sided IE are haemoptysis caused by septic pulmonary embolisms and acute right-heart failure due to tricuspid regurgitation [1,2]. Another systemic complication related to $S$. aureus infection is acute diffuse glomerulonephritis caused by immune complex formation and complement $\mathrm{C} 3$ deposits in the glomeruli [3-6]. Right-sided endocarditis is commonly associated with intravenous drug abuse, central venous catheters, and implantable cardiac device infections. However, patients with a congenital ventricular septal defect (VSD) are at high risk of IE [7-10]. Echocardiographic findings, such as a left-to-right shunt in IE, should always raise the suspicion of an acquired VSD, principally in previous young and healthy patients [10-14]. In the literature, several cases of left ventricular-to-right atrial shunt, also known as the Gerbode defect [15], were reported in association with IE [12-14, 16-18].

\section{Case presentation}

A 23-year-old man was referred from another hospital with a history of a dry cough, fever $\left(>39^{\circ} \mathrm{C}\right)$, and malaise, mostly at noon and at night. The symptoms started after he developed a self-limited skin and soft tissue infection on his left hand two weeks ago. Initially, he was hospitalized for 3 days under suspicion of COVID-19 infection. Empiric antibiotic therapy with piperacillin-tazobactam was started after his admission. Transthoracic echocardiography revealed floating vegetation $(35-40 \mathrm{~mm})$ on the tricuspid valve. Prior to transfer to our clinic, he also complained of blood-stained sputum and two episodes of diarrhoea and vomiting. The patient originally came from Romania, is a construction worker, and denied intravenous drug abuse. However, he admitted to having contact with a patient with active pulmonary tuberculosis in the past.

On physical examination at admission, his vital signs showed a blood pressure of $128 / 60 \mathrm{mmHg}$, a heart rate of $112 \mathrm{bpm}$, oxygen saturation of $97 \%$ on room air and a subfebrile temperature $\left(37.5^{\circ} \mathrm{C}\right)$. Cardiac auscultation revealed a grade III/VI holosystolic murmur over the tricuspid valve. There were also bilateral rales and crackles audible at the base of the lungs and right basal dullness on percussion. Examination of his extremities and skin revealed bilateral ankle pitting oedema and an isolated left-hand oedema with concomitant swelling of the third metacarpal-phalangeal joint. Neurological examination was unremarkable.

Initial investigations included laboratory tests and blood culture sets. An electrocardiogram showed a sinus rhythm without signs of underlying ischaemia or atrioventricular block. An initial full blood count revealed mild leucocytosis, neutrophilia, left deviation, and microcytic anaemia (Table 1). High levels of C-reactive protein and procalcitonin suggested a bacterial infection. In addition, the patient presented with a concomitant acute kidney injury (creatinine $1.5 \mathrm{mg} / \mathrm{dl}, \mathrm{BUN} 112 \mathrm{mg} /$ dl). Liver function tests showed elevated levels of alkaline phosphatase and gamma-GT and low levels of cholinesterase (Table 1). Empirical antibiotic therapy with ampicillin, flucloxacillin and gentamicin, according to the current European Guidelines for the empirical treatment of native valve endocarditis, was administered [1]. A throat swab for the SARS-CoV-2-RNA PCR test was reported to be negative.

After admission, we performed transoesophageal echocardiography (Fig. 1, Additional file 1: Video 1), which demonstrated $25 \times 15 \mathrm{~mm}$ vegetation on the septal leaflet of the tricuspid valve without evidence of severe tricuspid regurgitation. The other valves

Table 1 Initial laboratory investigations

\begin{tabular}{|c|c|c|}
\hline Test & Result & Normal range \\
\hline \multicolumn{3}{|l|}{ Full blood count (FBC) } \\
\hline Leucocyte count & $16,5401 / \mu l$ & $3800-10,300$ \\
\hline Erythrocyte count & $3.05 \mathrm{Mio} / \mu \mathrm{l}$ & $4.2-6.2$ \\
\hline Haemoglobin & $8.7 \mathrm{~g} / \mathrm{dl}$ & $14-18$ \\
\hline Haematocrit & $24.2 \%$ & $42-52$ \\
\hline $\mathrm{MCH}$ & $28.5 \mathrm{pg}$ & $27-34$ \\
\hline $\mathrm{MCHC}$ & $36.0 \mathrm{~g} / \mathrm{dl}$ & $32-36$ \\
\hline MCV & $79.3 \mathrm{fl}$ & $80-93$ \\
\hline Thrombocyte count & $211 \times 10^{9} / \mathrm{L}$ & $150-450$ \\
\hline C-reactive protein (CPR) & $21.24 \mathrm{mg} / \mathrm{dl}$ & $\max .0 .50$ \\
\hline Procalcitonin & $9.75 \mathrm{ng} / \mathrm{ml}$ & $\max .0 .1$ \\
\hline ESR 1st hour & $57 \mathrm{~mm}$ & $0-15$ \\
\hline Creatinine & $1.6 \mathrm{mg} / \mathrm{dl}$ & $0.6-1.1$ \\
\hline GFR-CKD-EPI & $60 \mathrm{ml} / \mathrm{min} / 1.73 \mathrm{~m}^{2}$ & $>60$ \\
\hline BUN & $112 \mathrm{mg} / \mathrm{dl}$ & $12-46$ \\
\hline Albumin & $1.3 \mathrm{~g} / \mathrm{dl}$ & $3.0-5.0$ \\
\hline \multicolumn{3}{|l|}{ Liver function tests (LFT) } \\
\hline AST/GOT & $40 \mathrm{U} / \mathrm{I}$ & $\max .50$ \\
\hline ALT/GPT & $35 \mathrm{U} / \mathrm{I}$ & $\max .50$ \\
\hline GGT & $138 \mathrm{U} / \mathrm{l}$ & $\max .60$ \\
\hline $\mathrm{LDH}$ & $207 \mathrm{U} / \mathrm{I}$ & $\max .250$ \\
\hline Alkaline phosphatase (ALP) & $138 \mathrm{U} / \mathrm{l}$ & $40-130$ \\
\hline Bilirubin total & $1.1 \mathrm{mg} / \mathrm{dl}$ & $\max .1 .1$ \\
\hline Cholinesterase (CHE) & $2.0 \mathrm{kU} / \mathrm{l}$ & $4.9-12.0$ \\
\hline
\end{tabular}

Mean corpuscular haemoglobin (MCH), mean corpuscular haemoglobin concentration (MCHC), mean corpuscular volume (MCV), erythrocyte sedimentation rate (ESR), glomerular filtration rate (GFR), blood urea nitrogen (BUN), aspartate amino transferase (AST), alanine amino transferase (ALT), gamma glutamyl transferase (GGT), lactate dehydrogenase (LDH) 


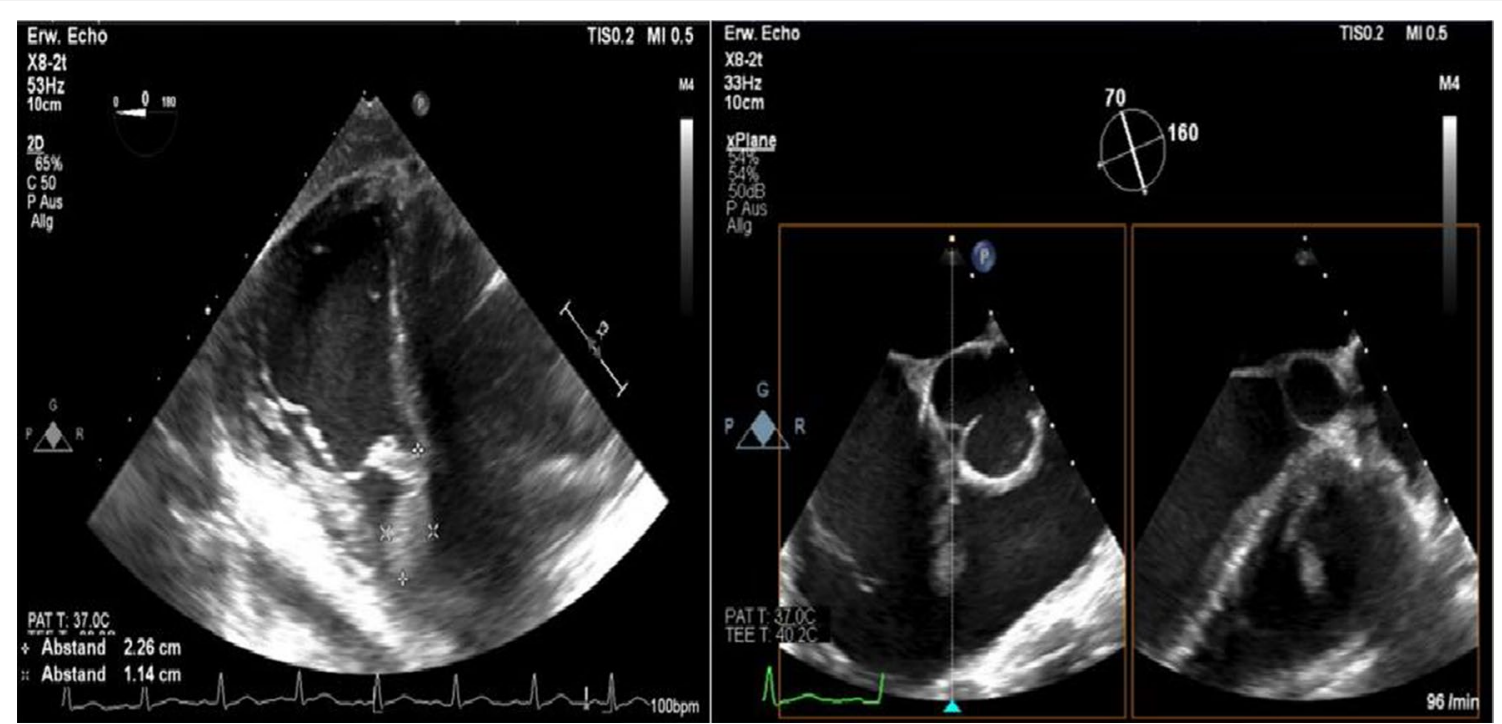

Fig. 1 Initial transoesophageal echocardiography showing vegetation on the septal leaflet of the tricuspid valve

presented no vegetation or severe regurgitation. The left ventricular ejection fraction was normal, and intracardiac thrombi were not observed. Further relevant echocardiographic findings revealed a normal right ventricular function and pulmonary arterial systolic pressure of $30 \mathrm{mmHg}$. A small ventricular left-to-right shunt due to a ventricular septal defect was observed on colour Doppler (Fig. 2, Additional file 1: Video 2).
In addition, a CT scan of the thorax and abdomen demonstrated bilateral disseminated multiple septic pulmonary emboli and concomitant pneumonic infiltrates as well as mild bilateral pleural effusions (Fig. 3). On the $\mathrm{CT}$ abdomen scan, no additional organ emboli or abscess formations were found. A coronary $\mathrm{CT}$ angiography showed no evidence of calcium plaques suggestive of coronary artery disease or malformations. Although this patient did not present neurological symptoms, a brain

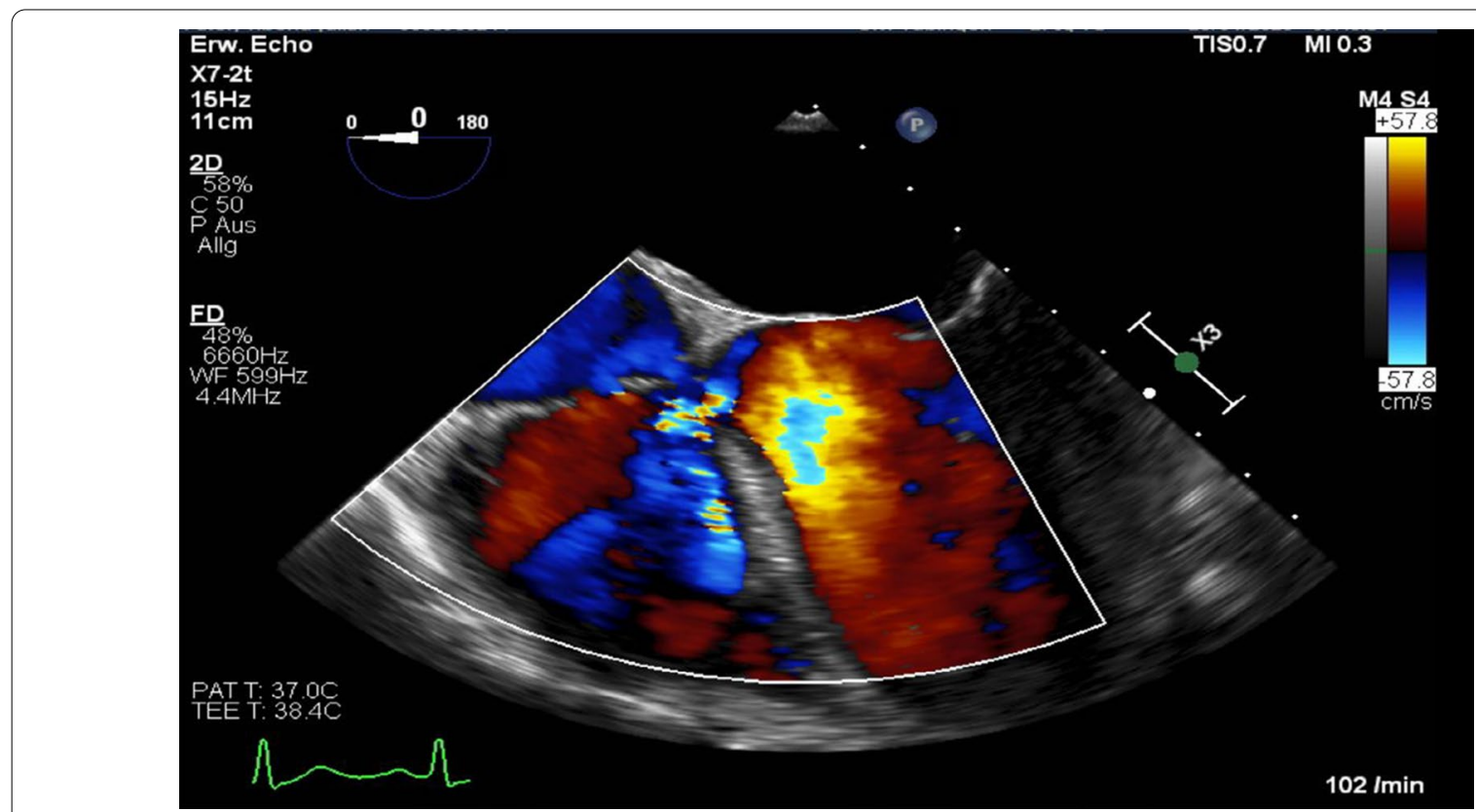

Fig. 2 Transoesophageal echocardiography showing a left-to-right shunt in colour Doppler corresponding to a VSD 


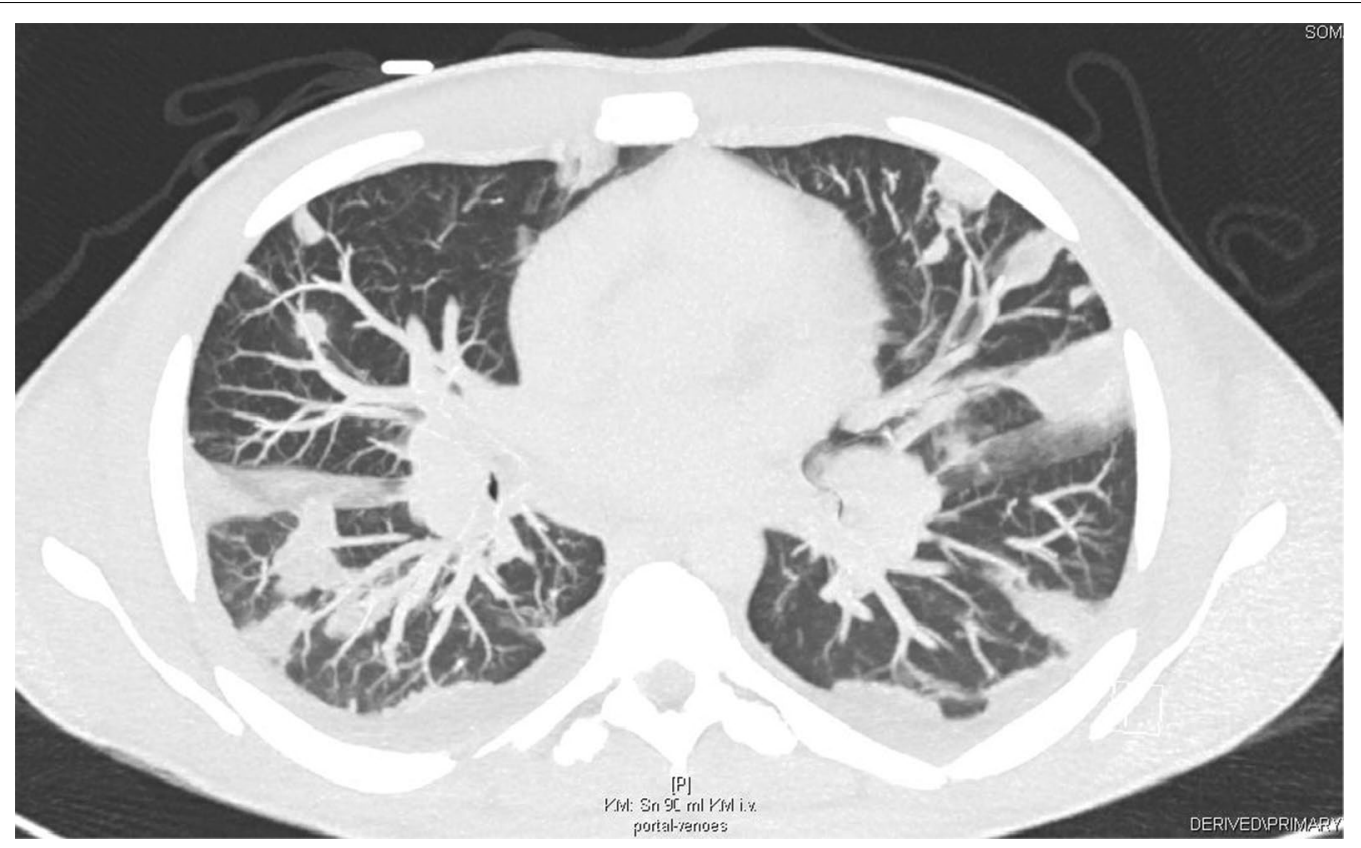

Fig. 3 A thorax CT scan demonstrating bilateral pneumonic infiltrates, septic emboli, and pleural effusions

CT was obtained, which showed normal findings. A CT scan of the left hand revealed a soft-tissue infection or phlegmon without bone involvement.

After admission, at least two blood culture sets were reported to be positive for methicillin-sensitive Staphylococcus aureus (MSSA). The final diagnosis of S. aureus bacteraemia and isolated right-sided endocarditis was based on two major criteria according to the modified Duke criteria for endocarditis [1]. We continued administering flucloxacillin, following the current guidelines [1]. Clarithromycin was started because of atypical pneumonic infiltrates on CT, and a course of 10 days of ceftazidime was also added to the antibiotic regimen due to concern about a Pseudomonas sp. infection. Previously, the initial antibiotic therapy with gentamicin was stopped in consideration of his acute kidney injury. A therapeutic intravenous anticoagulation with unfractionated heparin was begun after the diagnosis of septic pulmonary emboli.

Furthermore, a second transoesophageal echocardiography was performed 10 days after starting antibiotic therapy (Fig. 4). Fortunately, it showed diminished vegetation $(16 \times 7 \mathrm{~mm})$ on the septal leaflet of the tricuspid valve, without any evidence of further valve lesions. The left-to-right shunt due to the ventricular septal defect was stable without haemodynamic significance. In consensus with our endocarditis team, including a cardiac surgery evaluation, we decided on conservative management of the isolated right-sided endocarditis.
During hospitalization, the patient gained weight and there was an increase in peripheral oedema and anasarca, and increased levels of creatinine, BUN, and hypoalbuminemia were noted on laboratory tests (Table 1). In addition, urine diagnostic tests revealed macrohematuria, albuminuria, high levels of A1-microglobulin, and a high protein-creatinine ratio, suggesting acute tubular injury (Table 2). Autoantibodies and C3 complement tests were conducted, which revealed a low $\mathrm{C} 3$ level and a negative ANCA titre (Table 3). In consideration of these findings, we suspected glomerulonephritis associated with $S$. aureus infection and decided to not perform a renal biopsy. Instead, we administered diuretic therapy with amiloride and hydrochlorothiazide to treat the anasarca symptoms. In addition, relevant proteinuria was observed on his 24-h urine protein test (Table 2). Consequently, we started a regimen of corticosteroids with prednisolone therapy $(1 \mathrm{mg} / \mathrm{kg} \mathrm{BW})$ for 4 weeks, and then it was tapered weekly, showing a gradual improvement in the patient's proteinuria and oedema (Table 2).

After an acute episode of massive haemoptysis, we stopped the intravenous anticoagulation and performed an urgent bronchoscopy (Fig. 5). It showed tracheobronchitis with diffuse bleeding in segment 8 of the lower right lobe requiring an endobronchial tamponade for $24 \mathrm{~h}$ to stop the bleeding. Subsequently, the patient underwent a revision bronchoscopy for extraction of the endobronchial tamponade. It showed abundant purulent bronchial secretions without signs of de novo active 


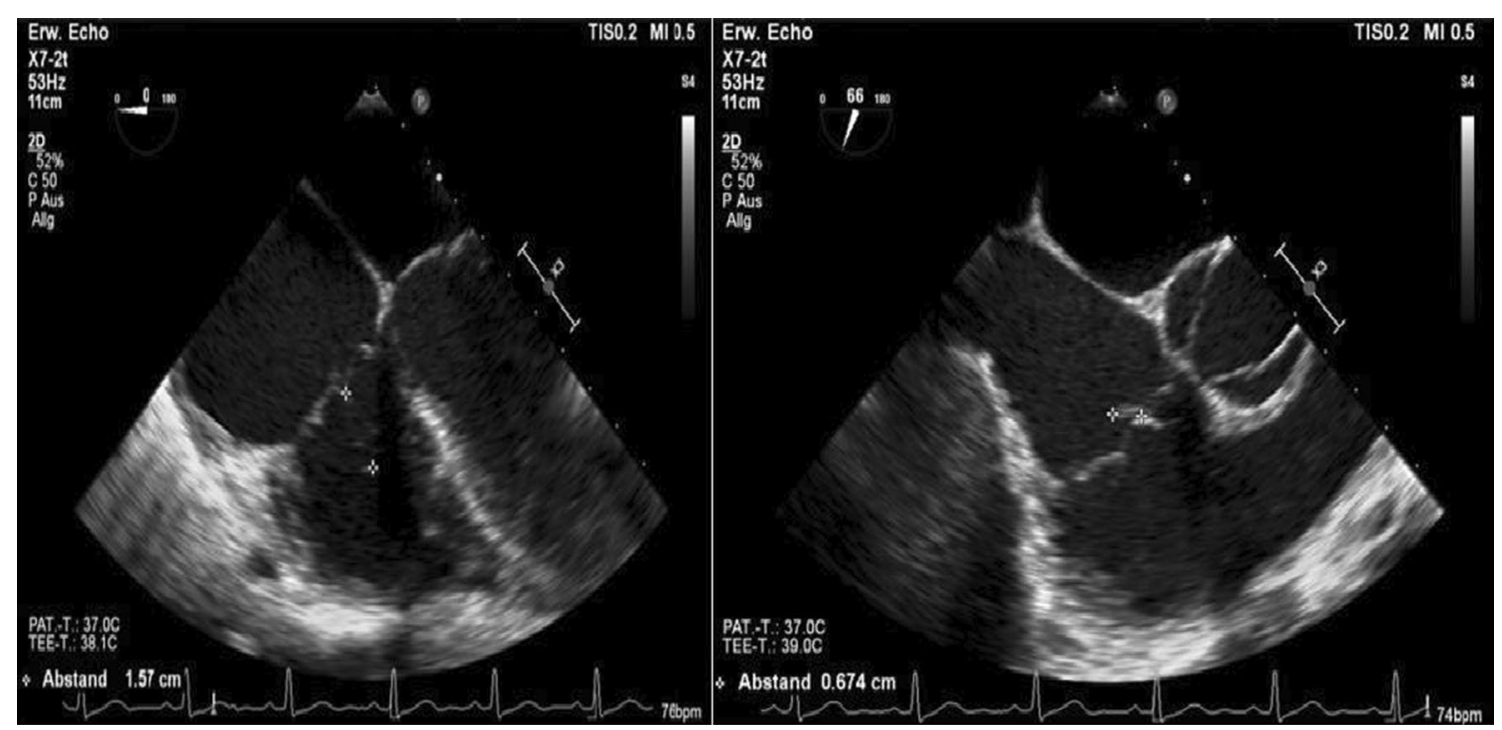

Fig. 4 Transoesophageal echocardiography showing a decrease in vegetation on the septal leaflet of the tricuspid valve

Table 2 Urine diagnostic tests

\begin{tabular}{llll}
\hline Test spot urine sample & On admission & At follow-up (4 weeks later) & Normal range, units \\
\hline Protein & 7.18 & 1.19 & $<0.10 \mathrm{~g} / \mathrm{L}$ \\
Creatinine & 86 & 158 & $\mathrm{mg} / \mathrm{dL}$ \\
Protein/creatinine ratio & 8349 & 753 & $<100 \mathrm{mg} / \mathrm{g}$ \\
Albumin & 4420 & 742 & $<20 \mathrm{mg} / \mathrm{L}$ \\
A1-microglobulin & 529 & 21 & $<13 \mathrm{mg} / \mathrm{L}$ \\
A1-microglobulin/creatinine ratio & 615.1 & 13.3 & $<13.0 \mathrm{mg} / \mathrm{g}$ \\
A2-macroglobulin & 12.5 & $<2.3$ & $<2.4 \mathrm{mg} / \mathrm{L}$ \\
IgG & 1850 & 127 & $<10 \mathrm{mg} / \mathrm{L}$ \\
IgG/creatinine ratio & 2151.2 & 80.4 & $<10 \mathrm{mg} / \mathrm{g}$ \\
$24-\mathrm{h}$ urine test & & & \\
Creatinine /24 h & 1221 & & $800-2000 \mathrm{mg} / 24 \mathrm{~h}$ \\
BUN /24 $\mathrm{h}$ & 16,724 & & $5500-22,000 \mathrm{mg} / 24 \mathrm{~h}$ \\
Protein /24 h & 9.95 & & $\mathrm{max} .0 .15 \mathrm{~g} / 24 \mathrm{~h}$ \\
Protein/creatinine ratio & 8152 & $\mathrm{max} .100 \mathrm{mg} / \mathrm{g}$ \\
A1-microglobulin & 105 & & $\mathrm{max} .13 \mathrm{mg} / \mathrm{L}$ \\
A2-macroglobulin & 15.1 & & $0-2.4 \mathrm{mg} / \mathrm{L}$ \\
Albumin /24 h & 5698 & & $\mathrm{max} .30 \mathrm{mg} / 24 \mathrm{~h}$ \\
\hline
\end{tabular}

Immunoglobulin G (lgG), blood urea nitrogen (BUN)

bleeding. Acid-fast stain tests, Pneumocystis jirovecii, and respiratory viruses (RSV-RNA, Influenza A, B) were negative in the bronchoalveolar lavage. Although the gamma-interferon test was positive for a latent tuberculosis infection, we considered it an isolated finding not relevant to diagnose active pulmonary tuberculosis. Haemoptysis episodes are more likely due to multiple septic pulmonary emboli and concomitant $S$. aureus pneumonia, as previously found on the patient's CT-thorax scans.

Further investigations related to explaining the microcytic anaemia revealed an iron deficiency (iron $33 \mu \mathrm{g} /$ $\mathrm{dL}$, ferritin $116 \mathrm{mcg} / \mathrm{dL}$, transferrin $78 \mathrm{mg} / \mathrm{dL}$ ). However, this finding might be explained as a combination of nutrition deficiency, recurrent haemoptysis, and inflammatory systemic disease due to acute infective endocarditis. His 


\section{Table 3 Autoantibodies tests}

\begin{tabular}{ll}
\hline Test & Result (normal range) \\
\hline Anti-GMB antibodies & $3.1 \mathrm{U} / \mathrm{ml}(<7)$ \\
ANA & $1:<80 \mathrm{U} / \mathrm{ml}$, negative \\
ANCA & $1:<10 \mathrm{U} / \mathrm{ml}$, negative \\
CANCA, pANCA & $80 \mathrm{mg} / \mathrm{dl}(90-180)$ \\
C3-Complement & $14 \mathrm{mg} / \mathrm{dl}(10-40)$ \\
C4-Complement &
\end{tabular}

Antinuclear antibody (ANA), anti-neutrophil cytoplasmic antibody (ANCA), antiglomerular basement membrane antibodies (anti-GMB) other parameters were at normal levels without suspicion of haemolysis or vitamin B12 deficiency.

Prior to discharge, a thorax CT scan revealed remission of the pneumonic infiltrates and septic emboli after two weeks of antibiotic therapy (Fig. 6). After 4 weeks of hospitalization, considerable improvement of renal function, proteinuria, and inflammatory markers was observed. The patient was discharged with combined antibiotic therapy of flucloxacillin and clarithromycin at 4 weeks.

At follow-up, one month after discharge, the patient presented with a good recovery of renal function and

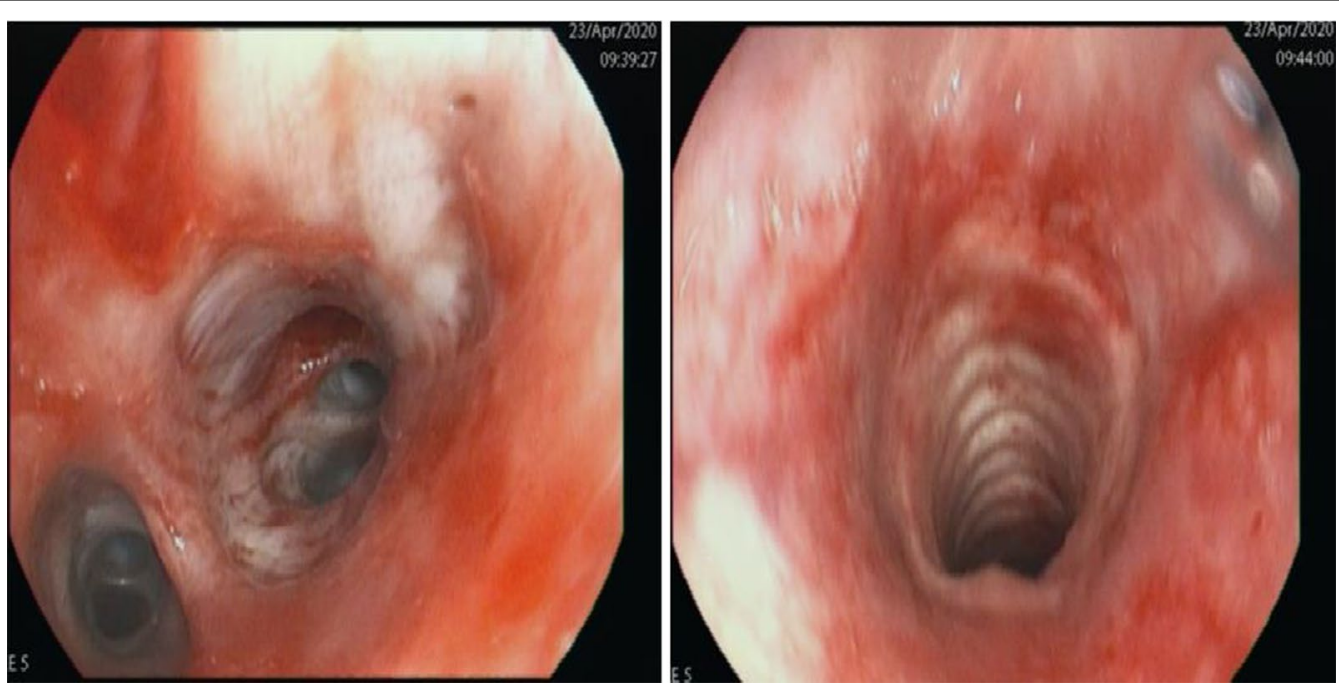

Fig. 5 Bronchoscopy showing tracheobronchitis and diffuse bleeding of the lower right lobe

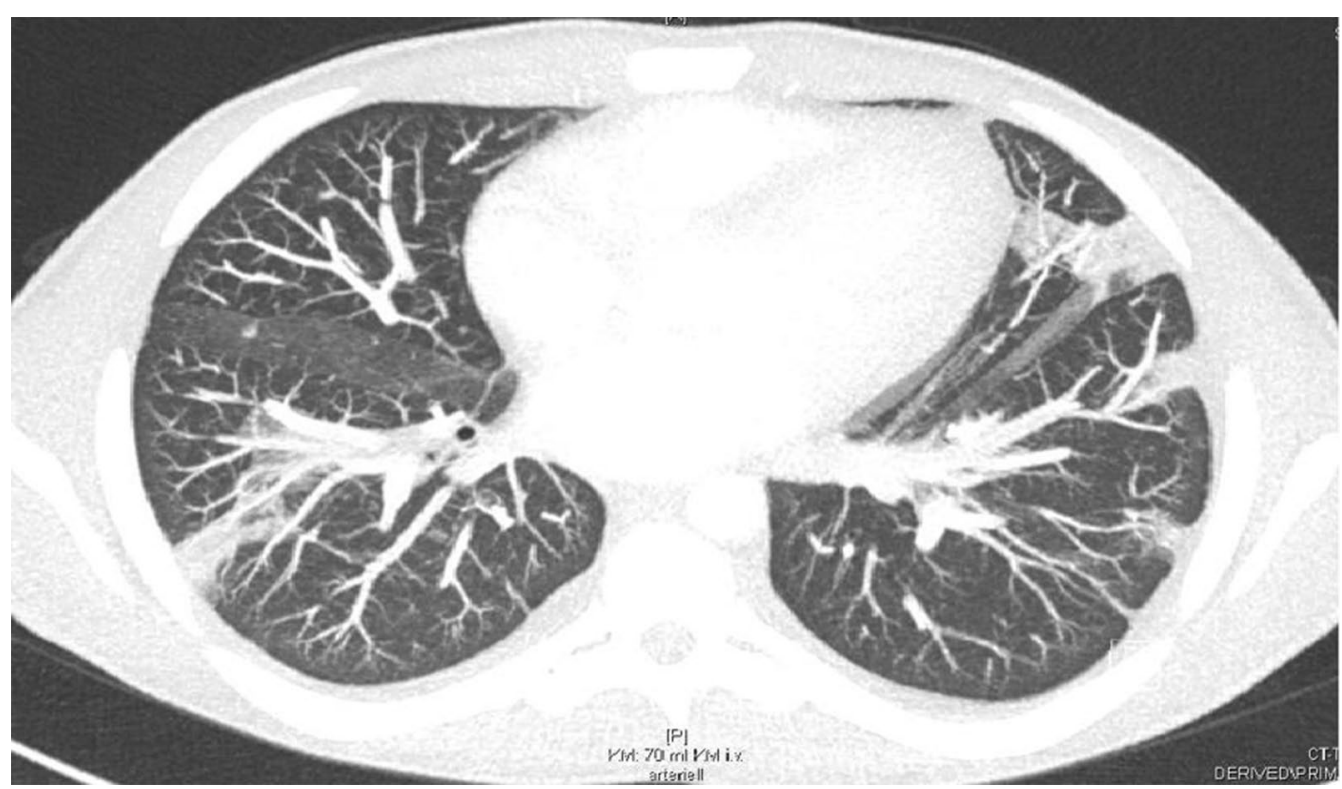

Fig. 6 A follow-up thorax CT scan demonstrated fewer pneumonic infiltrates and septic pulmonary emboli 
proteinuria (Table 2). Laboratory tests were unremarkable. Additionally, transoesophageal echocardiography showed no more relevant vegetation of the tricuspid valve only some mild regurgitation (Fig. 7). However, an aneurysmal transformation of the ventricular septal defect $(5 \mathrm{~mm})$ located infravalvular to the septal leaflet of the tricuspid valve was found (Fig. 8, Additional file 1: Video 3). The left-to-right shunt was not haemodynamically significant (systolic velocity of $5.5 \mathrm{~m} / \mathrm{s}$ ) without involvement of the aortic valve or aorta (Fig. 9). After surgical evaluation, conservative management, including endocarditis prophylaxis, was continued. The patient was scheduled for further echocardiographic follow-up and cardiac catheterization to determine the invasive haemodynamic parameters.

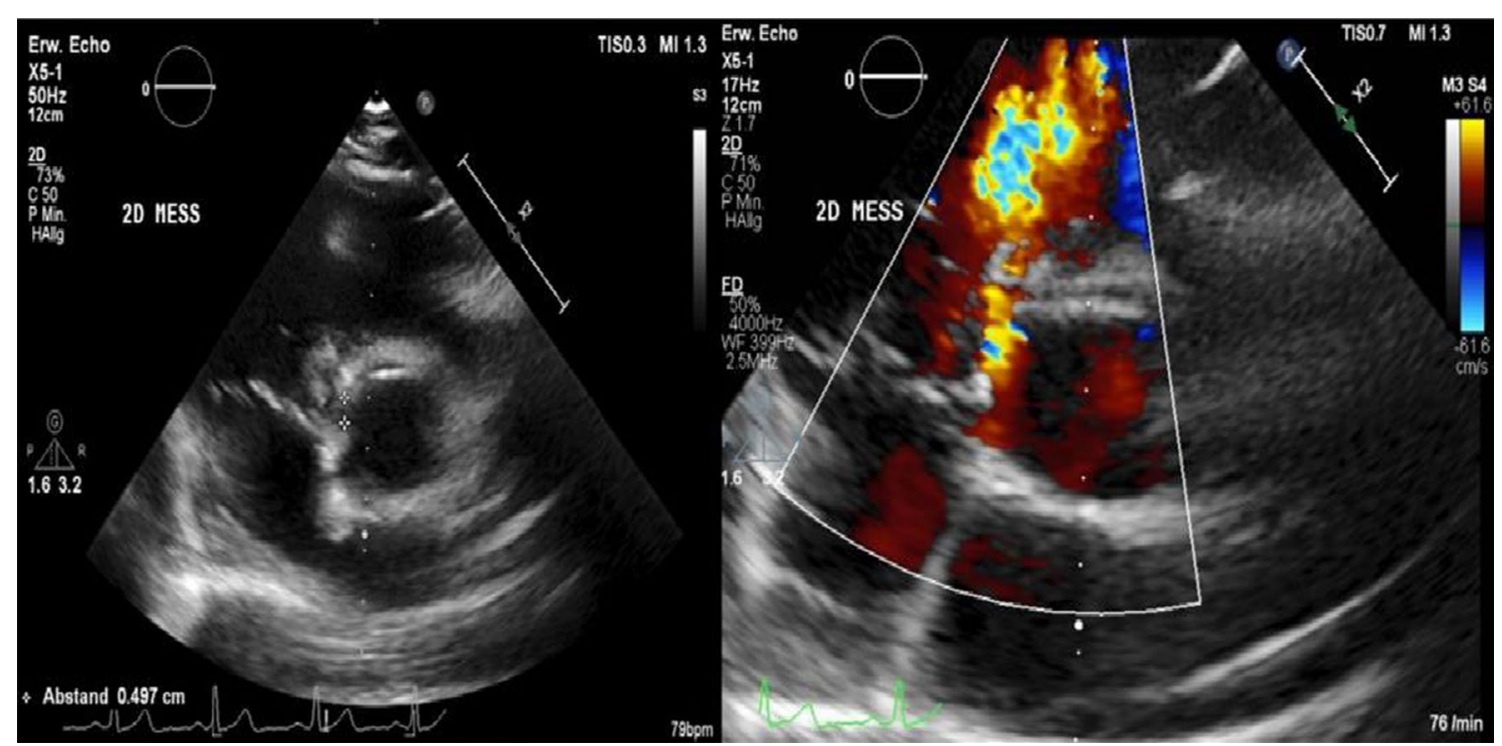

Fig. 7 Transoesophageal echocardiography at the 4-week follow-up showed a $5 \mathrm{~mm}$ VSD and a left-to-right shunt

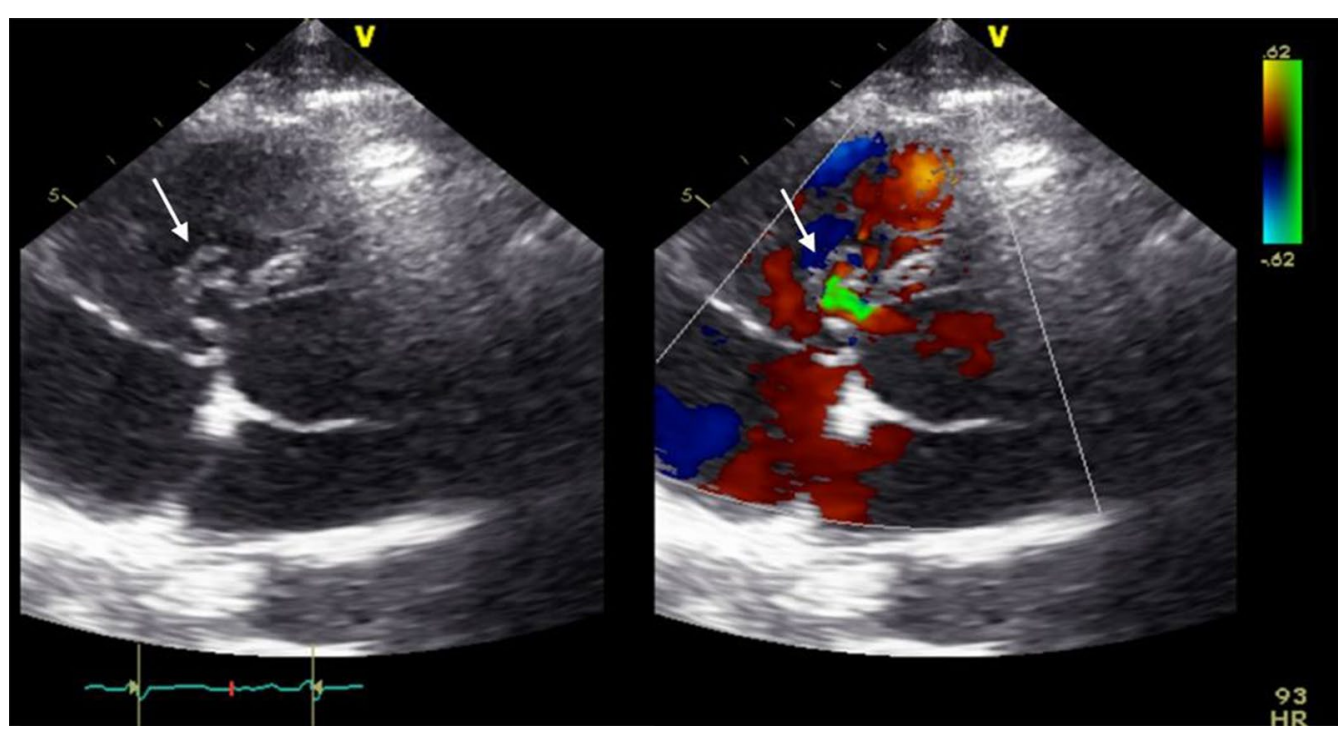

Fig. 8 Aneurysmal transformation of the VSD with involvement of the septal leaflet of the tricuspid valve 


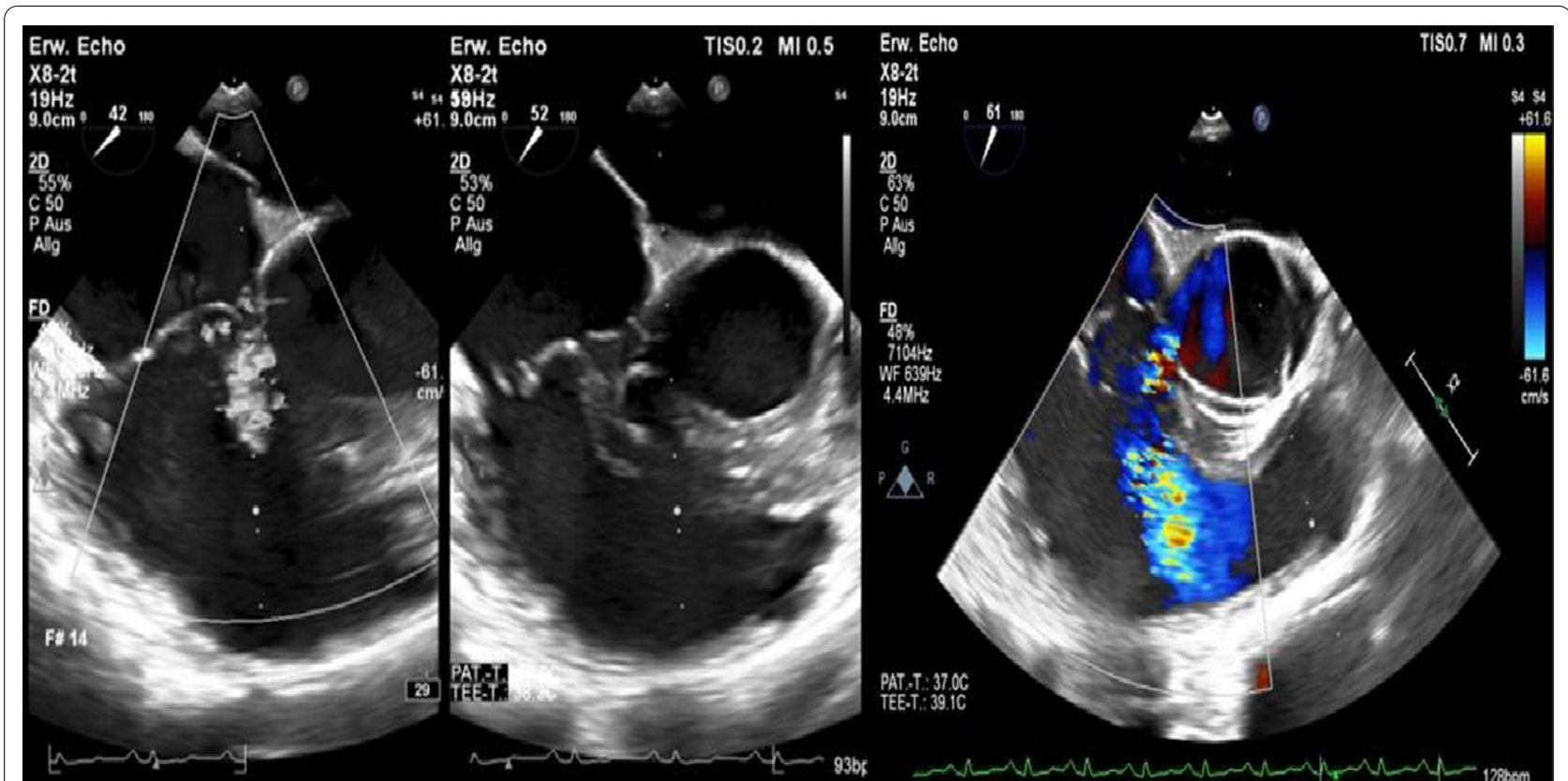

Fig. 9 Transoesophageal echocardiography showing a VSD corresponding to a type 2 Gerbode defect

\section{Discussion and conclusions}

Ventricular septal defects (VSDs) with left-to-right ventricular shunts are frequently congenital and are associated with a higher incidence of endocarditis in comparison with patients without congenital ventricular septal defects [7-10]. Acquired VSD after an episode of endocarditis has been previously described in several case reports as a very rare complication [12-14, 16-18]. The Gerbode defect is a perimembranous VSD with a secondary left ventricular-to-right atrium shunt $[10,15]$.
Gerbode was an American cardiac surgeon who successfully reported the first surgical management of five patients with VSD and left-to-right atrial shunt in 1958 [15].

Classical features of the Gerbode defect are communication between the left ventricle and the right atrium through a ventricular septal defect localized supra- or infravalvular in anatomical relation to the septal leaflet of the tricuspid valve $[10,15,19]$. According to the classification described by Perry et al. [19], a type 1 Gerbode

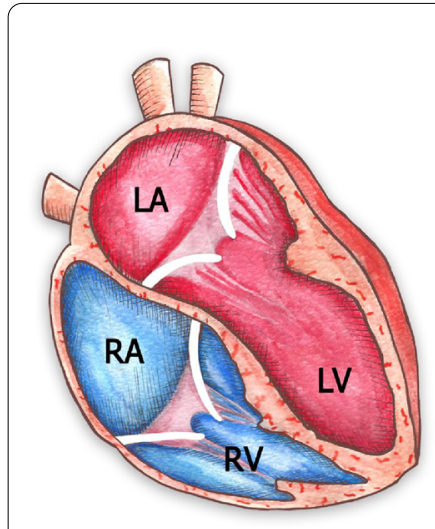

a Normal

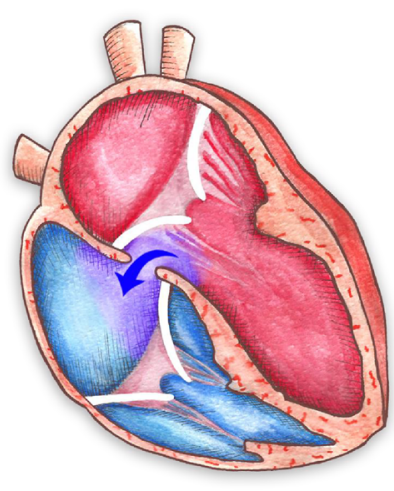

b Type 1 Supravalvular (Direct)

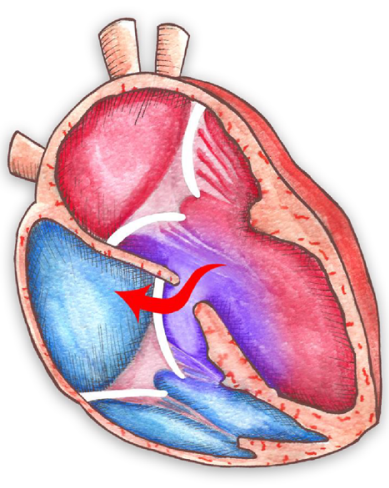

c Type 2 Infravalvular (Indirect)

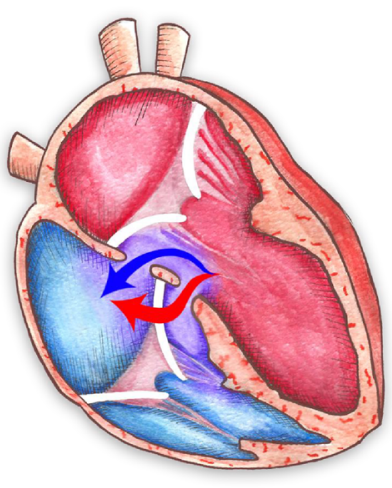

d Type 3 Intermediate

Fig. 10 Classification and anatomical features of the 3 types of the Gerbode defect. (Illustration by Ivonne Hernández del Muro @e 2020) 
defect consists of a left-ventricular-to-right-atrium shunt localized supravalvular to the tricuspid valve (Fig. 10). In contrast, in type 2 Gerbode defects, there is a left-ventricular-to-right-ventricular shunt localized infravalvular to the septal leaflet of the tricuspid valve, and owing to tricuspid regurgitation, an indirect left-ventricular-toright atrium communication develops (Fig. 10). Type 3 Gerbode defects consist of a combination of both supraand infravalvular left-to right shunts (Fig. 10) [10, 19].

The diagnosis of a ventricular septal defect is based on clinical and echocardiographic findings, such as evidence of cardiac murmurs in association with a left-toright ventricular shunt on colour Doppler that may be difficult to detect in asymptomatic young patients, who might unknowingly be at a higher risk of endocarditis [7, 9]. Skin and soft tissue infections, as reported in this case, represent a portal of bacterial entry to the blood circulation, causing $S$. aureus endocarditis. However, current European and American guidelines do not routinely recommend endocarditis prophylaxis in patients with acyanotic congenital heart defects, since this population is considered at intermediate risk for infective endocarditis $[1,20]$.

Infective endocarditis, specifically caused by $S$. aureus, has been related to other common complications, such as nephrotic syndrome and glomerulonephritis [3-6]. The underlying pathologic mechanism is immune-mediated due to the formation of immune complexes and glomerular deposition of complement $\mathrm{C} 3[3,5,6]$. In this case, we decided to administer corticosteroid therapy to treat acute diffuse glomerulonephritis secondary to $S$. aureus infection in combination with antibiotic therapy with a satisfactory reduction of proteinuria and gradual improvement of the patient's renal function.

Finally, ventricular septal defects complicated by endocarditis without haemodynamic significance are commonly treated conservatively with endocarditis prophylaxis to avoid further endocarditis episodes. Successful surgical management of small ventricular septal defects without haemodynamic significance after endocarditis has been reported in several cases [10, 21-24].

Right-sided infective endocarditis in patients with unknown ventricular septal defects has relevant clinical significance, from diagnosis to management, intervention, and the prevention of further endocarditis episodes. An acquired ventricular septal defect after tricuspid endocarditis is very rare, but it has been described as a possible complication [12-14, 16-18]. Patients with known congenital ventricular septal defects should also be included as high-risk patients in the guidelines to receive appropriate endocarditis prophylaxis. Moreover, severe complications of right-sided endocarditis, including septic pulmonary embolisms with a frequent incidence of haemoptysis and concomitant pneumonia, might require invasive interventions, intensive care management, and mechanical ventilation. Nephrotic syndrome and glomerulonephritis due to acute $S$. aureus infection is a common complication of infective endocarditis $[3,5,6]$. Concomitant antibiotic and corticosteroid therapy might be required to improve proteinuria and renal function, as we described in this case.

In conclusion, patients with right-sided endocarditis often have common risk factors. However, screening for ventricular septal defects is mandatory in patients with a negative history of intravenous drug abuse, implantable cardiac devices, or central venous catheter infections. Current guidelines do not recommend endocarditis prophylaxis in acyanotic heart defects. However, patients with congenital ventricular septal defects are at high risk for infective endocarditis after common bacterial exposure, such as dental procedures and soft-tissue infections, which require endocarditis prophylaxis.

An acquired ventricular septal defect after endocarditis is a very rare complication, but there are many clinical cases reported in the previous literature. Therefore, echocardiographic follow-up is mandatory in all cases. Complications of infective endocarditis should be managed within an interdisciplinary team to reduce morbidity and improve outcomes. Surgical treatment of postendocarditis ventricular septal defects is still controversial in cases without haemodynamic significance.

\section{Supplementary information}

Supplementary information accompanies this paper at https://doi. org/10.1186/s12872-020-01772-y.

Additional file 1. Video 1: Transoesophageal echocardiography showing vegetation on the septal leaflet of the tricuspid valve. Video $\mathbf{2}$ : Transoesophageal echocardiography showing 378 a left-to-right shunt corresponding to atype 2 Gerbode defect. Video 3: Transoesophageal echocardiography showing a type 2 Gerbode defect after tricuspid valve endocarditis.

\section{Abbreviations}

IE: Infective endocarditis; VSD: Ventricular septal defect; COVID-19: Coronavirus disease-19; FBC: Full blood count; $\mathrm{MCH}$ : Mean corpuscular haemoglobin; MCHC: Mean corpuscular haemoglobin concentration; MCV: Mean corpuscular volume; CPR: C-reactive protein; ESR: Erythrocyte sedimentation rate; GFR: Glomerular filtration rate; BUN: Blood urea nitrogen; AST: Aspartate amino transferase; ALT: Alanine amino transferase; GGT: Gamma glutamyl transferase; LDH: Lactate dehydrogenase; CT: Computer tomography; MSSA: Methicillinsensitive Staphylococcus aureus; BW: Body weight; IgG: Immunoglobulin G; ANA: Antinuclear antibody; ANCA: Anti-neutrophil cytoplasmic antibody; Anti-GMB: Anti-glomerular basement membrane antibodies; RSV: Respiratorysyncytial virus.

\section{Acknowledgements}

We especially thank our colleagues at the Echocardiography Laboratory of the Department of Cardiology and Angiology, University Hospital Tuebingen and the University of Tuebingen for their support. We acknowledge support by Open Access Publishing Fund of University of Tübingen. We especially 
thank Ivonne Hernández del Muro, Medical Illustrator, National Autonomous University of Mexico, for her illustration used in this publication.

\section{Authors' contributions}

GM analysed and interpreted the patient echocardiography findings and laboratory tests during hospitalization and at follow-up. RSH interpreted all patient data, obtained the patient's consent, conducted a literature research and review and was a major contributor to writing the main manuscript. All authors read and approved the final manuscript.

\section{Funding}

Open Access funding enabled and organized by Projekt DEAL. Institutional Funding of the University of Tuebingen, Germany.

\section{Availability of data and materials}

All data generated or analysed during this study are included in this published article

\section{Ethics approval and consent to participate}

An ethics approval for this case report was not necessary.

\section{Consent for publication}

Written informed consent was obtained from the patient for the publication of this case report and any accompanying images. A copy of the written consent is available for review by the Editor of this journal.

\section{Competing interests}

The authors declare that they have no competing interests.

Received: 15 August 2020 Accepted: 8 November 2020

Published online: 23 November 2020

\section{References}

1. Habib G, Lancellotti P, Antunes MJ, Bongiorni MG, Casalta JP, Del Zotti F, et al. Eur Heart J. 2015;36:3075-128.

2. Baddour LM, Wilson WR, Bayer AS, Fowler VG Jr, Tleyjeh IM, Rybak MJ, et al. Infective endocarditis in adults: diagnosis, antimicrobial therapy, and management of complications: a Scientific Statement for Healthcare Professionals from the American Heart Association. Circulation. 2015:132:1435-86.

3. Boils CL. Endocarditis-associated glomerulonephritis. In: Satoskar AA, Nadasdy T, editors. Bacterial infections and the kidney. Cham: Springer; 2017. p. $87-116$

4. Tu WH, Shearn MA, Lee JC. Acute diffuse glomerulonephritis in acute staphylococcal endocarditis. Ann Intern Med. 1969;71:335-41.

5. Neugarten J, Gallo GR, Baldwin DS. Glomerulonephritis in bacterial endocarditis. Am J Kidney Dis. 1984;3:371-9.

6. Pulik M, Lionnet F, Genet P, Petitdidier C, Vacher B. Immune-complex glomerulonephritis associated with Staphylococcus aureus infection of a totally implantable venous device. Support Care Cancer. 1995;3:324-6.

7. Berglund $E$, Johansson B, Dellborg M, Sörensson P, Christersson C, Nielsen $\mathrm{NE}$, et al. High incidence of infective endocarditis in adults with congenital ventricular septal defect. Heart. 2016;102:1835-9.

8. Shah P, Singh WS, Rose V, Keith JD. Incidence of bacterial endocarditis in ventricular septal defects. Circulation. 1966:34:127-31.
9. Wu MH, Wang JK, Lin MT, Wu ET, Lu FL, Chiu SN, et al. Ventricular septal defect with secondary left ventricular-to-right atrial shunt is associated with a higher risk for infective endocarditis and a lower late chance of closure. Pediatrics. 2006;117:e262-7.

10. Taskesen T, Prouse AF, Goldberg SL, Gill EA. Gerbode defect: another nail for the 3D transesophagel echo hammer? Int J Cardiovasc Imaging. 2015;31:753-64.

11. Battin M, Fong LV, Monro JL. Gerbode ventricular septal defect following endocarditis. Eur J Cardiothorac Surg. 1991:5:613-4.

12. Velebit V, Schöneberger A, Ciaroni S, Bloch A, Maurice J, Christenson JT, et al. "Acquired" left ventricular-to-right atrial shunt (Gerbode defect) after bacterial endocarditis. Tex Heart Inst J. 1995;22:100-2.

13. Prifti E, Ademaj F, Baboci A, Demiraj A. Acquired Gerbode defect following endocarditis of the tricuspid valve: a case report and literature review. J Cardiothorac Surg. 2015;10:115.

14. Gonçalves AM, Correia A, Falcão LM. Endocardite da válvula tricúspide em doente com cardiopatia congénita. Rev Port Cardiol. 2013;32:53-8.

15. Gerbode F, Hultgren H, Melrose D, Osborn J. Syndrome of left ventricularright atrial shunt; successful surgical repair of defect in five cases, with observation of bradycardia on closure. Ann Surg. 1958;148:433-46.

16. Nayyar M, King B, Garg N. When guidelines fail, a case study in infective endocarditis and perimembranous ventral septal defect. J Am Coll Cardiol. 2016;67:1016.

17. Xhabija N, Prifti E, Allajbeu I, Sula F. Gerbode defect following endocarditis and misinterpreted as severe pulmonary arterial hypertension. Cardiovasc Ultrasound. 2010;8:44.

18. Kretzer A, Amhaz H, Nicoara A, Kendall M, Glower D, Jones MM. A case of gerbode ventricular septal defect endocarditis. CASE (Phila). 2018;2:207-9.

19. Perry EL, Burchell HB, Edwards JE. Congenital communication between the left ventricle and the right atrium; co-existing ventricular septal defect and double tricuspid orifice. Proc Staff Meet Mayo Clin. 1949:24:198-206.

20. Wilson W, Taubert KA, Gewitz M, Lockhart PB, Baddour LM, Levison M, et al. Prevention of infective endocarditis: guidelines from the American Heart Association: a guideline from the American Heart Association Rheumatic Fever, Endocarditis, and Kawasaki Disease Committee, Council on Cardiovascular Disease in the Young, and the Council on Clinical Cardiology, Council on Cardiovascular Surgery and Anesthesia, and the Quality of Care and Outcomes Research Interdisciplinary Working Group. Circulation. 2007;116:1736-54.

21. Tavakkoli Hosseini M, Quarto C, Bahrami T. Quadruple-valve infective endocarditis and ventricular septal defect. Tex Heart Inst J. 2013:40:209-10.

22. L'Ecuyer TJ, Embrey RP. Closure of hemodynamically insignificant ventricular septal defect after infective endocarditis. Am J Cardiol. 1993;72:1093-4.

23. Oakley GD, Carson PH, Sanderson JM. Right-sided endocarditis involving both tricuspid and pulmonary valves in a patient with ventricular septal defect. Br Heart J. 1977;39:323-5.

24. Mellins RB, Cheng G, Ellis K, Jameson AG, Malm JR, Blumenthal S. Ventricular septal defect with shunt from left ventricle to right atrium. Bacterial endocarditis as a complication. Br Heart J. 1964;26:584-91.

\section{Publisher's Note}

Springer Nature remains neutral with regard to jurisdictional claims in published maps and institutional affiliations. 